\title{
Novel balloon-and-aspiration method for cerebral venous sinus thrombosis: dental-floss technique
}

\author{
Yoshikazu Matsuda, MD,,2 Hideo Okada, MD,1,3 Joonho Chung, MD, PhD, 1,4 \\ R. Webster Crowley, MD, ${ }^{1}$ and Demetrius K. Lopes, MD1
}

\begin{abstract}
'Department of Neurological Surgery, Rush University Medical Center, Chicago, Illinois; ${ }^{2}$ Department of Neurosurgery, Wakayama Medical University; ${ }^{3}$ Department of Neurosurgery, Wakayama Rosai Hospital, Wakayama City, Japan; and ${ }^{4}$ Department of Neurosurgery, Gangnam Severance Hospital, Yonsei University College of Medicine, Seoul, Republic of Korea
\end{abstract}

\begin{abstract}
Cerebral venous sinus thrombosis is sometimes fatal. The standard treatment for sinus thrombosis is anticoagulation, but endovascular intervention must be considered when medical treatment fails. Mechanical thrombectomy is usually required when a large clot burden exits. Unfortunately, in sinus thrombosis attributable to a clot burden larger than that in an intracranial artery, the conventional technique used for intraarterial acute stroke intervention with a stent retriever and/ or aspiration is not very effective. The authors describe here their endovascular approach to mechanical thrombectomy for sinus thrombosis using aspiration combined with angioplasty balloon support.
\end{abstract}

https://thejns.org/doi/abs/10.3171/2017.1.FOCUS16519

KEY WORDS cerebral venous sinus thrombosis; balloon catheter; aspiration catheter; novel technique

$\mathrm{D}$ ESPITE our improved understanding and diagnosis of sinus thrombosis, its resulting morbidity and mortality rates are still high. ${ }^{6}$ Standard medical treatment of sinus thrombosis typically consists of heparin in the acute phase and eventual conversion to low-molecular-weight heparin or other anticoagulant medications. ${ }^{2}$ However, this treatment is controversial for patients with symptomatic intracranial hemorrhage, because anticoagulation confers risk for further hemorrhage expansion. In addition, sinus thromboses can often be refractory to anticoagulation therapy, particularly in patients with complete sinus occlusion. In these patients, circulating heparin can come in contact with the polar ends of a clot, without penetrating the clot in between, and obviate much of its desired effect. For patients in whom medical management has failed, mechanical thrombectomy is a good alternative.

There have been reports of endovascular treatment involving infusion of thrombolytic agents, ${ }^{12}$ balloon angioplasty, ${ }^{7,13}$ and, more recently, the combination of a stent retriever and aspiration. ${ }^{5}$

We describe here the "venous sinus floss" technique for recanalization of acute sinus thrombosis. Three cases are described to illustrate this concept.

\section{Operative Technique}

The venous sinus floss technique requires arterial (5Fr catheter) and venous (8-Fr guide catheter or 6-Fr guide sheath) vascular access. Intravenous heparin is given during the procedure so that the activated clotting time is kept between 250 and 350 seconds. An arterial diagnostic catheter $(5 \mathrm{Fr})$ is placed in the vessel that generates the best roadmap of the venous anatomy. The venous access is "parked" ideally closer to the area of sinus occlusion. An aspiration catheter, usually an ACE64 (Penumbra), is advanced over a TransForm balloon catheter (Stryker) and a Synchro 14 microguidewire (Stryker), although the aspiration catheter can also be tracked over a 0.035 -inch guidewire if the thrombus is particularly dense. The balloon and microguidewire are navigated carefully distal to the occluded sinus. Depending on the length of the thrombosed sinus, the use of an exchange-length microguidewire might be preferred. The distal tip of the wire is advanced as far as possible, which enables the balloon to move back and forth across the entire segment of interest without moving the wire. For example, for a transverse sinus thrombus that involves the torcula, the wire can be placed from one 
internal jugular vein to another. Once the wire is in place distally, the balloon is inflated and dragged back toward the aspiration catheter without moving the wire, while continuous aspiration is applied to the catheter. After reaching the aspiration catheter, the balloon is advanced again over the wire to a point distal to the thrombus and moved back and forth in a flossing movement. The aspiration catheter can be advanced to the interface of the clot, or even through much of the clot, to shorten the distance that the balloon is tracked. This procedure might prevent the thrombus from occluding previously uninvolved cortical veins, and it provides the added benefit of some direct clot aspiration independent of the flossing maneuver.

\section{Illustrative Cases \\ Case 1}

This male teenager had a history of acute lymphocytic leukemia and sinus venous thrombosis since 2013. He was admitted to an outside hospital with a diagnosis of West Nile encephalitis. The following day, he developed rightsided weakness and seizures. Subsequent head CT imaging revealed multiple small bihemispheric focal hemorrhages (Fig. 1A), and the patient was transferred to our hospital for further care. The patient's NIH Stroke Scale score was 5 on admission. Repeated head CT scanning with angiography and venography revealed a nonocclusive filling defect of the superior sagittal sinus and thrombosis in frontoparietal superficial cortical veins bilaterally (Fig. 1B). Intravenous heparin was initiated, and the patient was observed in the neurological intensive care unit. Two days later, he developed difficulty with speech and increased right-sided weakness. Head CT revealed progression of his left-sided hemorrhage (Fig. 1C). Because of his progressive hemorrhage while on medical management, the anticoagulation therapy was stopped, and he was brought in for endovascular treatment.

The patient was brought to the angiography suite and given general anesthesia. The right common femoral artery was selectively catheterized using a 5-Fr sheath, as was the right common femoral vein using an 8-Fr sheath. A right internal carotid artery angiogram revealed the thrombus in the superior sagittal sinus without occlusion (Fig. 2A). An 8-Fr Neuron Max 088 catheter (Penumbra) was advanced into the right transverse-sigmoid junction.
An ACE64 aspiration catheter and a $4 \times 15-\mathrm{mm}$ TransForm balloon catheter were advanced successfully into the area of occlusion over a Synchro wire. After the balloon was advanced distal to the thrombus, it was inflated. The inflated balloon was moved several times along the microguidewire from one side of the thrombus to the other while aspiration was applied to the ACE64 catheter (Fig. 2B). Final angiography revealed clearance of the thrombus and excellent venous drainage (Fig. 2C).

By the following day, motor strength in the patient's right lower extremity had improved but his arm weakness, dysarthria, and loss of fluency remained. Repeat MR venography performed before discharge revealed a continued decrease in clot burden. Ten days after treatment, the patient was discharged to an acute rehabilitation hospital with an NIH Stroke Scale score of 10.

\section{Case 2}

This woman in her 4th decade, with no significant medical history, was admitted to an outside hospital 1 week after delivering a child; she had experienced an episode of loss of consciousness with severe headache. Imaging revealed thrombosis of the superior sagittal sinus, and treatment with intravenous heparin was initiated. Two days after admission, she complained of severe worsening of her headache followed by decreased responsiveness. Repeat imaging revealed extension of the thrombus down to the bilateral transverse sinuses and right jugular vein, at which point she was transferred to our hospital for endovascular treatment.

The patient was brought to the angiography suite, and general anesthesia was induced. The right common femoral artery was selectively catheterized using a 5-Fr sheath, as was the right common femoral vein using an 8-Fr sheath. Bilateral internal carotid artery and left vertebral artery angiography revealed occlusion of all deep and superficial venous drainage. An 8-Fr Neuron Max 088 catheter was advanced into the right jugular vein. An ACE64 aspiration catheter and a TransForm $7 \times 15-\mathrm{mm}$ balloon catheter over a Synchro microguidewire were successfully advanced distal to the clot in all directions, and the flossing maneuver was used by moving the inflated balloon back and forth over the microguidewire while aspiration was applied to the ACE64. This process was

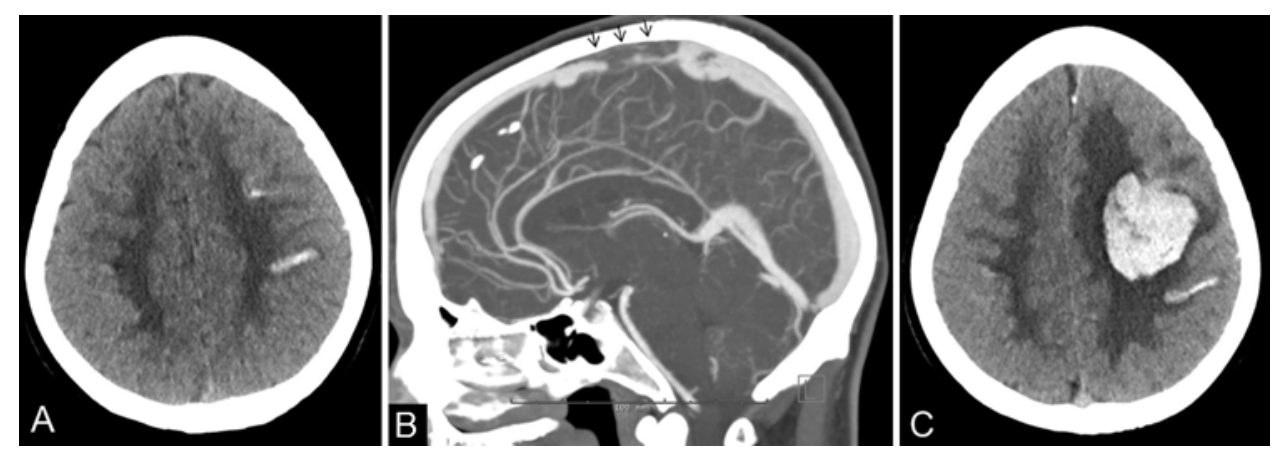

FIG. 1. Case 1. Head CT and CT angiographic images. A: Axial head CT scan showing multiple bilateral small focal hemorrhages. B: Sagittal CT angiogram demonstrating a thrombus in the superior sagittal sinus (arrows). C: Axial CT scan showing an increase in the left hemorrhage. 

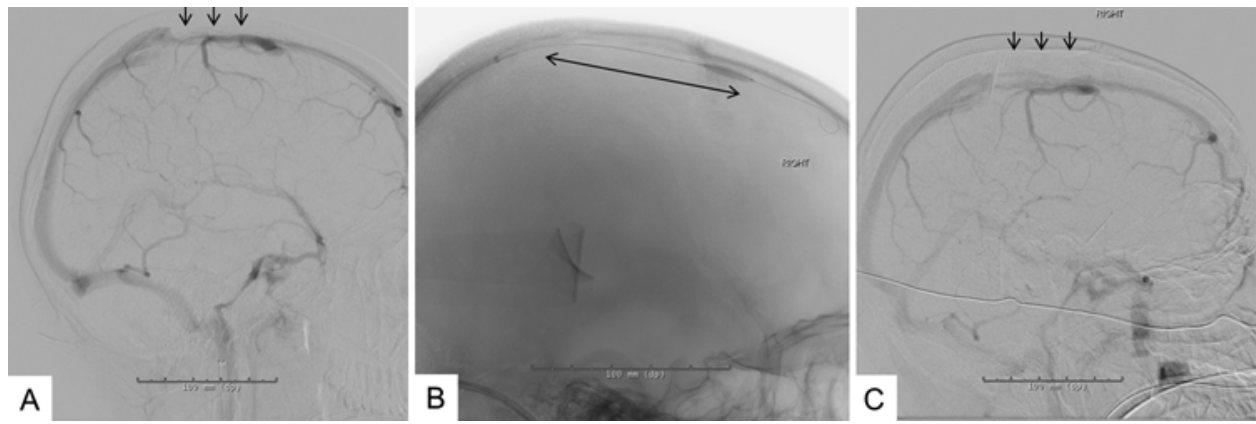

FIG. 2. Case 1. Angiographic images. A: Lateral digital subtraction angiogram before the procedure showing partial defect of the superior sagittal sinus (arrows). B: Unsubtracted lateral angiogram showing the Penumbra ACE64 aspiration catheter and inflated TransForm $4 \times 15-\mathrm{mm}$ balloon catheter in the superior sagittal sinus. The balloon is being moved back and forth (double arrow). C: Lateral digital subtraction angiogram after the procedure demonstrating improvement of the defect in the superior sagittal sinus (arrows).

performed initially from the superior sagittal sinus back to the right jugular vein but was also performed for the inferior sagittal sinus and the contralateral transverse sinus. Final angiography revealed improved recanalization with some residual thrombus.

After the procedure, the patient was noted to have right gaze deviation. MRI revealed a right frontal venous infarct and left parietal venous infarct with a small amount of subarachnoid hemorrhage. She eventually showed substantial improvement with only subtle neglect of the left side (which had resolved by the time of subsequent examinations) and unsteady gait. She was transferred to an acute rehabilitation hospital 15 days after the procedure.

\section{Case 3}

This female teenager had a significant medical history of Crohn disease and use of prednisone and oral contraceptive pills. She had presented with a 1-week history of severe headache and vomiting. Head CT scanning and CT angiography performed at an outside hospital revealed extensive venous sinus thrombosis extending into the superior and inferior sagittal sinus, the straight sinus, and bilateral transverse sinuses. She was started on intravenous heparin and subsequently transferred to our institution for a higher level of care. On arrival, she was drowsy but neurologically intact. However, shortly thereafter, she developed weakness and numbness in her right lower extremity. MRI demonstrated left frontoparietal infarction (Fig. 3). After her weakness worsened, she was prepared for mechanical thrombectomy.

The patient was brought to the angiography suite, and general anesthesia was induced. The right common femoral artery was selectively catheterized using a 5-Fr sheath, as was the right common femoral vein using an 8-Fr sheath. Angiography revealed a defect of contrast among the superior sagittal sinus, transverse sinus, sigmoid sinus, and straight sinus with no obvious sinus drainage (Fig. 4A). A Neuron Max 088 guide catheter was advanced up to the right jugular bulb. An ACE64 catheter over the 0.035 -inch guidewire was advanced through the clot of the right transverse sinus and up into the superior sagittal sinus until it was approximately in the anterior third of the superior sagittal sinus. A TransForm $4 \times 15-\mathrm{mm}$ balloon catheter over a Synchro guidewire was advanced as far as possible distally and inflated in the anterior third of the superior sagittal sinus. The ACE64 catheter was brought back several centimeters under aspiration to increase the distance between the balloon and the catheter, and the balloon was pulled back and forth to the ACE64 slowly in a flossing maneuver. The same procedure was repeated sequentially from the superior sagittal sinus to the right sigmoid sinus and straight sinus (Fig. 4B and C). Final angiography demonstrated substantially improved flow through the superior sagittal sinus down through the right transverse sinus and down to the right jugular bulb. In addition, flow to the straight sinus was seen (Fig. 4D).

Intravenous heparin was continued after the thrombectomy. The following day, bifrontal hemorrhages on her CT scan consistent with hemorrhagic conversion of venous infarctions were noted, and neurological examination revealed continued worsening of her condition. She eventually developed refractory intracranial hypertension that required decompressive craniectomy, and she died 4 days later.

\section{Discussion}

The mortality rate of cerebral venous thrombosis has
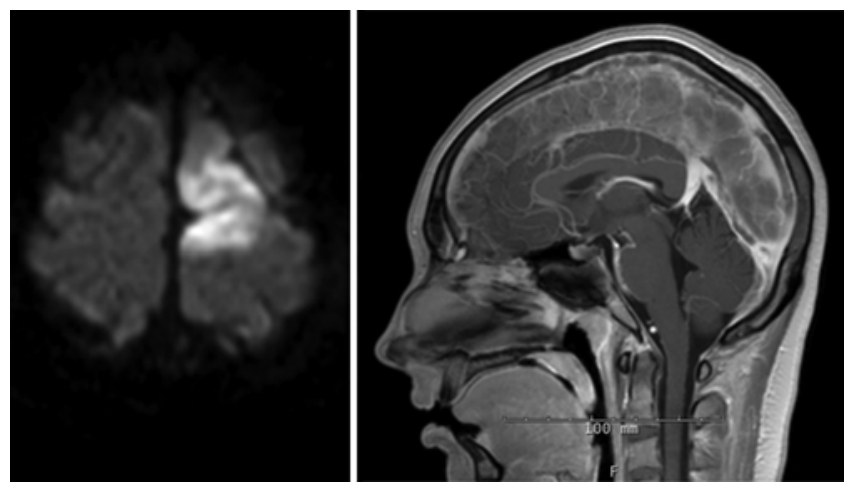

FIG. 3. Case 3. MR images. Left: Diffusion-weighted image showing a left-sided frontoparietal infarction. Right: Sagittal contrast-enhanced T1-weighted image revealing diffuse thrombus in the superior sagittal sinus and straight sinus. 


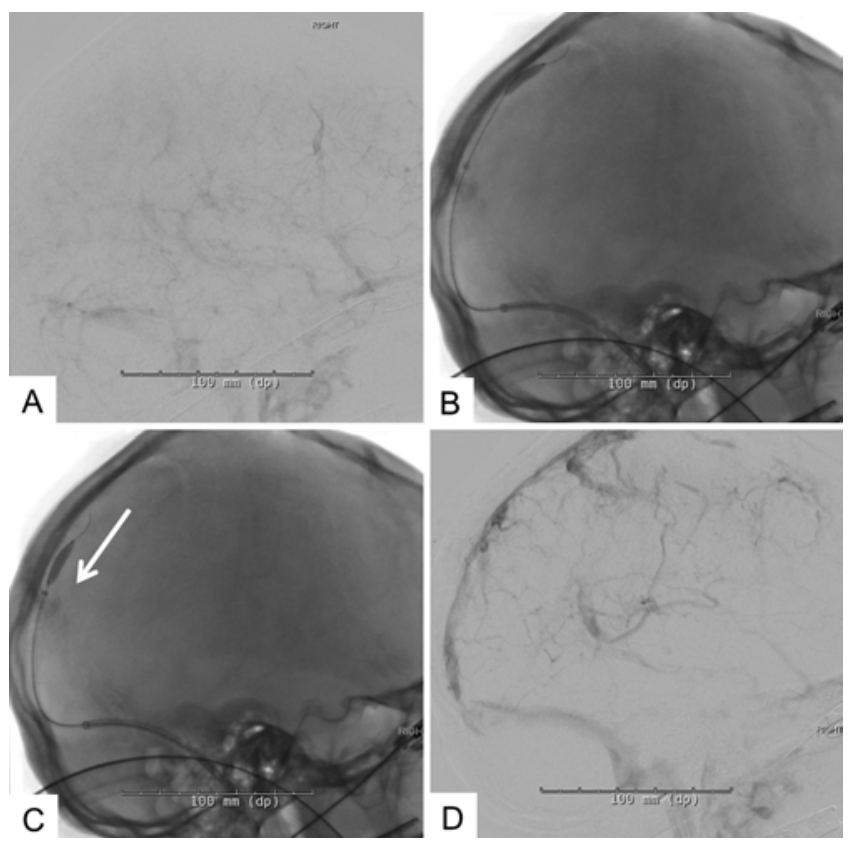

FIG. 4. Case 3. Angiographic images. A: Lateral digital subtraction angiogram showing a defect of contrast in the superior sagittal sinus, transverse sinus, sigmoid sinus, and straight sinus with no obvious sinus drainage. B: Lateral unsubtracted angiogram demonstrating the inflated balloon and aspiration catheter in the posterior part of the superior sagittal sinus. C: Lateral unsubtracted angiogram showing the inflated balloon pulling back to the aspiration catheter in the posterior part of the superior sagittal sinus (arrow). D: Final lateral digital subtraction angiogram revealing improvement of drainage from the superior sagittal sinus to the sigmoid sinus and straight sinus.

decreased from $40 \%-50 \%$ in the 1960 s to $0 \%-28 \%$ after 2000. It might be closer to $5 \%,{ }^{6,9}$ but in 2015 , Mokin et al. ${ }^{11}$ reported that the mortality rate in their multicenter experience was $27 \%$. This rate is not negligible.

Standard medical treatment for sinus thrombosis is heparinization with conversion to low-molecular-weight heparin or oral anticoagulant agents. ${ }^{2}$ However, for certain patients with a refractory condition or for those who have a higher risk of bleeding, anticoagulation might be either futile or too risky. After failed medical treatment, endovascular treatment is generally considered the next option for the treatment of sinus thrombosis. Several reports have described endovascular treatment using techniques such as balloon angioplasty, ${ }^{7,13}$ stenting, ${ }^{8}$ use of a microsnare $^{1}$ or stent retriever, ${ }^{9}$ and catheter aspiration. ${ }^{5}$ Endovascular thrombolysis is performed by introducing a thrombolytic enzyme, such as urokinase or recombinant tissue plasminogen activator, directly into the clot through catheterization. ${ }^{12}$ However, the risk of intracranial bleeding has limited the use of these thrombolytic enzymes. El Tecle et al. ${ }^{7}$ reported venoplasty with a Scepter XC balloon catheter (MicroVention) for sinus thrombosis followed by tissue plasminogen activator infusion. Mokin et al. ${ }^{11}$ reported that a Penumbra aspiration catheter was used in $69 \%$ of all cases after the larger-bore aspiration catheters (5MAX and 5MAX ACE) were introduced. Successful mechanical thrombectomy with the Solitaire FR device (Medtronic) was also reported recently. ${ }^{9}$ However, a larger clot burden might make recanalization more likely to fail. As has been found with treatment of acute arterial largevessel occlusion, a combined technique with aspiration is being performed more commonly; Mokin et al. reported that multimodality treatment was performed in $62 \%$ of their cases. A combination of the AngioJet system (Boston Scientific) and a Penumbra catheter has been reported to be potentially useful for sinus thrombosis; however, a length discrepancy between the devices necessitated cutting the Penumbra 5MAX catheter. ${ }^{3}$ Mascitelli et al..$^{10}$ also reported a combined technique with a stent retriever and Penumbra catheter as the stent anchor with mobile aspiration technique.

The venous sinus floss technique is performed by moving an inflated balloon back and forth along a microguidewire from a point distal to the thrombus to a point proximal to the occluded sinus repeatedly while providing continuous aspiration. As a result, a thrombus in the sinus can be disrupted or pulled closer to the aspiration catheter so that recanalization can be achieved. Actually, in the present 3 cases, we failed to remove clots using the previously reported methods. We judged that clots removed by the dental-floss technique were so hard that previous methods were unsuccessful. An additional advantage of this technique is that a larger balloon, such as one with a 7-mm diameter, can be used, so clots that might not be captured with catheter aspiration alone can be fragmented. We use a compliant balloon, because angioplasty is not needed. The balloon is used as a separator to create a channel through the clot while aspirating from the intermediate catheter. We also judged that this process is likely safer than using a noncompliant balloon, because the size of the occluded sinus is difficult to evaluate.

In their study, Brockmann et al. ${ }^{4}$ measured the diameter of the superior sagittal sinus on CT angiography and reported that its mean horizontal diameters were 6.7 and $8.6 \mathrm{~mm}$ and its mean vertical diameters were 5.2 and 6.4 $\mathrm{mm}$ at the level of the coronal suture and $3 \mathrm{~cm}$ dorsal to the coronal suture, respectively. Surgeons should inflate the balloon carefully with these findings in mind.

One concern with this procedure is that a thrombus can migrate to the pulmonary artery if there is a larger thrombus that is not aspirated successfully by the aspiration catheter, although there is probably a similar risk with other combined techniques. As a result, pulmonary embolism might occur, and consideration for this complication should be given.

The efficacy of this technique will need to continue to be investigated and possibly compared with that of existing strategies. Further experience is necessary.

\section{Conclusions}

In this technical report, we have described our initial experience with a venous sinus floss technique for sinus thrombosis. This technique might be an option for the endovascular treatment of sinus thrombosis.

\section{Acknowledgments}

We thank Kiffon Keigher for obtaining patient data. 


\section{References}

1. Bagley LJ, Hurst RW, Galetta S: Teener James, Sinson P: Use of a microsnare to aid direct thrombolytic therapy of dural sinus thrombosis. AJNR Am J Neuroradiol 170:784-786, 1998

2. Bentley JN, Figueroa RE, Vender JR: From presentation to follow-up: diagnosis and treatment of cerebral venous thrombosis. Neurosurg Focus 27(5):E4, 2009

3. Bress A, Hurst R, Pukenas B, Smith M, Kung D: Mechanical thrombectomy for cerebral venous sinus thrombosis employing a novel combination of Angiojet and Penumbra ACE aspiration catheters: the triaxial Angiojet technique. J Clin Neurosci 31:196-198, 2016

4. Brockmann C, Kunze S, Scharf J: Computed tomographic angiography of the superior sagittal sinus and bridging veins. Surg Radiol Anat 33:129-134, 2011

5. Choulakian A, Alexander MJ: Mechanical thrombectomy with the Penumbra system for treatment of venous sinus thrombosis. J Neurointerv Surg 2:153-156, 2010

6. Coutinho JM, Zuurbier SM, Stam J: Declining mortality in cerebral venous thrombosis: a systematic review. Stroke 45:1338-1341, 2014

7. El Tecle NE, Patel BM, El Ahmadieh TY, Daou MR, Hamade YJ, Zammar SG, et al: Novel use of a double lumen balloon catheter for venous sinus thrombolysis and venoplasty. $\mathbf{J}$ Clin Neurosci 22:1018-1020, 2015

8. Hunt MG, Lee AG, Kardon RH, Lesley WS, Chaloupka JC: Improvement in papilledema and visual loss after endovascular stent placement in dural sinus thrombosis. Neuroophthalmology 26:85-92, 2001

9. Kim H, Kim SR, Park IS, Kim YW: Treatment of the superior sagittal sinus thrombosis with the mechanical thrombectomy using stent-retriever device. J Korean Neurosurg Soc 59:518-520, 2016

10. Mascitelli JR, Pain M, Zarzour HK, Baxter P, Ghatan S,
Mocco J: Sinus thrombectomy for purulent cerebral venous sinus thrombosis utilizing a novel combination of the Trevo stent retriever and the Penumbra ACE aspiration catheter: the stent anchor with mobile aspiration technique. J Neurointerv Surg 8:e24, 2016

11. Mokin M, Lopes DK, Binning MJ, Veznedaroglu E, Liebman KM, Arthur AS, et al: Endovascular treatment of cerebral venous thrombosis: contemporary multicenter experience. Interv Neuroradiol 21:520-526, 2015

12. Patel SI, Obeid H, Matti L, Ramakrishna H, Shamoun FE: Cerebral venous thrombosis: current and newer anticoagulant treatment options. Neurologist 20:80-88, 2015

13. Yamashita S, Matsumoto Y, Tamiya T, Kawanishi M, Shindo A, Nakamura T, et al: Mechanical thrombolysis for treatment of acute sinus thrombosis-case report. Neurol Med Chir (Tokyo) 45:635-639, 2005

\section{Disclosures}

The authors report no conflict of interest concerning the materials or methods used in this study or the findings specified in this paper.

\section{Author Contributions}

Conception and design: Lopes. Acquisition of data: Matsuda, Crowley. Analysis and interpretation of data: Matsuda. Drafting the article: Matsuda. Critically revising the article: Lopes, Crowley. Reviewed submitted version of manuscript: Okada, Chung, Lopes.

\section{Correspondence}

Demetrius K. Lopes, Department of Neurological Surgery, Rush University Medical Center, 1725 W Harrison St., Professional Bldg., Ste. 855, Chicago, IL 60612. email: demetrius_lopes@ rush.edu. 